\title{
Spatial Attentional Bias as a Marker of Genetic Risk, Symptom Severity, and Stimulant Response in ADHD
}

\author{
Mark A Bellgrove*,1,2, Edwina Barry ${ }^{3}$, Katherine A Johnson ${ }^{2,3}$, Marie Cox ${ }^{3}$, Aoife Dáibhis,3, Michael Daly,3, \\ Ziarih Hawi ${ }^{3}$, David Lambert ${ }^{3}$, Michael Fitzgerald ${ }^{3}$, Fiona McNicholas ${ }^{4}$, lan H Robertson ${ }^{2}$, Michael Gill ${ }^{3}$ and \\ Aiveen Kirley \\ 'Cognitive Neuroscience Laboratory, School of Psychology and Queensland Brain Institute, University of Queensland, Brisbane, Qld., Australia; \\ ${ }^{2}$ School of Psychology and Trinity College Institute of Neuroscience, Trinity College Dublin, Dublin, Ireland; ${ }^{3}$ School of Medicine and Health \\ Sciences and Trinity College Institute of Neuroscience, Trinity College Dublin, Dublin, Ireland; ${ }^{4}$ Department of Child and Adolescent Psychiatry, \\ Our Lady's Hospital for Sick Children, Crumlin, Dublin, Ireland
}

\begin{abstract}
Attention-deficit hyperactivity disorder (ADHD) is a heritable childhood onset disorder that is marked by variability at multiple levels including clinical presentation, cognitive profile, and response to stimulant medications. It has been suggested that this variability may reflect etiological differences, particularly, at the level of underlying genetics. This study examined whether an attentional phenotypespatial attentional bias could serve as a marker of symptom severity, genetic risk, and stimulant response in ADHD. A total of 96 children and adolescents with ADHD were assessed on the Landmark Task, which is a sensitive measure of spatial attentional bias. All children were genotyped for polymorphisms ( $3^{\prime}$ untranslated (UTR) and intron 8 variable number of tandem repeats (VNTRs)) of the dopamine transporter gene (DATI). Spatial attentional bias correlated with ADHD symptom levels and varied according to DATI genotype. Children who were homozygous for the 10-repeat allele of the DATI 3'-UTR VNTR displayed a rightward attentional bias and had higher symptom levels compared to those with the low-risk genotype. A total of 26 of these children who were medication naive performed the Landmark Task at baseline and then again after 6 weeks of stimulant medication. Left-sided inattention (rightward bias) at baseline was associated with an enhanced response to stimulants at 6 weeks. Moreover, changes in spatial bias with stimulant medications, varied as a function of DATI genotype. This study suggests an attentional phenotype that relates to symptom severity and genetic risk for ADHD, and may have utility in predicting stimulant response in ADHD.

Neuropsychopharmacology (2008) 33, 2536-2545; doi: I 0.1038/sj.npp. I 30 I637; published online 28 November 2007
\end{abstract}

Keywords: ADHD; attention; dopamine; genetics; stimulants; DATI

\section{INTRODUCTION}

Attention-deficit hyperactivity disorder (ADHD) is a heritable childhood onset disorder with negative adult outcomes. Like many complex disorders, ADHD is marked by significant variability across a number of levels, including clinical presentation, cognitive profile, and response to stimulant medications; this variability may partly reflect differences in underlying genetics. Allelic variation in a number of candidate genes linked to catecholamine signaling is thought to confer risk for the disorder. The 10-repeat allele of a variable number of tandem repeat (VNTR) polymorphism within the dopamine transporter gene (DAT1) has attracted much interest as a

*Correspondence: Dr MA Bellgrove, School of Psychology and Queensland Brain Institute, University of Queensland, Brisbane, Queensland 4072, Australia, Tel: +61 73365 6516, Fax: +61 7 3365 4466, E-mail: m.bellgrove@uq.edu.au

Received 25 July 2007; revised 18 October 2007; accepted 27 October 2007 susceptibility allele for ADHD. The a priori hypothesis arises principally from the pharmacology of stimulant medications, such as methylphenidate (MPH), which inhibit the action of the transporter. A number of studies suggest that DAT1 is a gene of minor effect for ADHD with an average odds ratio of around 1.2 (Brookes et al, 2006; Cook et al, 1995; Daly et al, 1999; Gill et al, 1997). More recently, a number of studies have attempted to link allelic variation in DAT1 to intermediate constructs (Bellgrove et al, 2005a, b; Loo et al, 2003), or endophenotypes, and to both clinical (Kirley et al, 2003) and physiological measures of stimulant response (Gilbert et al, 2006; Loo et al, 2003). These studies could provide important collateral evidence regarding the functional effects of risk variants and highlight the potential clinical utility of molecular genetics for identifying subpopulations of children with ADHD that might respond better or more poorly to stimulant medication. Here, we report an association between a neurocognitive phenotypespatial attentional bias and DAT1 genotype in 96 children and adolescents with ADHD. We also show that spatial bias relates to symptom severity and is modified, as a function of 
DAT1 genotype, by stimulant medication. Finally, we show that independent of genotypic influences, spatial bias is a significant predictor of the clinical response to stimulant medications, such as $\mathrm{MPH}$, in medication naive children with ADHD.

The DAT1 $3^{\prime}$ untranslated (UTR) VNTR is potentially functional with a number of studies reporting an influence of 10-repeat variants either on in vitro measures of gene expression (Fuke et al, 2001; VanNess et al, 2005) or in vivo transporter densities (Cheon et al, 2005; Heinz et al, 2000). The dopamine transporter is the main site of action of stimulant medications, such as $\mathrm{MPH}$, which inhibit the reuptake of extra cellular dopamine by the transporter (Volkow et al, 1998). Both children and adults with ADHD have been reported to have increased DAT densities, in for example, the striatum and basal ganglia (Cheon et al, 2003; Dougherty et al, 1999; Dresel et al, 2000), but the evidence is not conclusive (Jucaite et al, 2005; Volkow et al, 2007). A recent and well-controlled PET study found increased DAT binding specifically in the right caudate of treatment naive adults with ADHD compared with matched control participants (Spencer et al, 2007). Stimulants have also been shown to reduce elevated dopamine transporter densities in adults with ADHD (Dresel et al, 2000; Krause et al, 2000; Vles et al, 2003).

Not surprisingly, a number of groups have investigated whether the 10-repeat DAT1 allele might mediate the clinical response achieved with stimulants. The results of these studies have proved inconclusive thus far with the 10repeat allele being associated with an enhanced (Kirley et al, 2003; Stein et al, 2005) or diminished (Cheon et al, 2005; Rohde et al, 2003; Roman et al, 2002; Winsberg and Comings, 1999) response to stimulants. Other studies, however, have failed to find an influence of alleles at the VNTR on stimulant response (Langley et al, 2005; Mick et al, 2006; van der Meulen et al, 2005). A number of studies have also reported interactions between DAT1 genotype and stimulants when using a physiological measure of brain function, such as an EEG frequency band, as an outcome variable (Gilbert et al, 2006; Loo et al, 2003). Important innovations within this field include the use of prospective pharmacogenetic designs with participants who are stimulant-medication naive at intake and the use of multiple predictors - both clinical and neuropsychological-of stimulant response.

Spatial attention refers to the perceptual enhancement of stimuli that are presented at certain locations in space. In most circumstances, healthy observers show a subtle bias of attention in favor of stimuli or events presented to the left, relative to right, side of space (Bradshaw, 1989). This lateral bias of attention to the left is thought to reflect the dominance of right-hemisphere neural networks for spatial processes; while right hemisphere networks direct attention to both left and right hemifields, left hemisphere networks do so only for the right hemifield (Mesulam, 1981). The dominance of the right-hemisphere for spatial attention is exemplified by the syndrome of unilateral neglect. Here, dysfunction in any one of the number of right hemisphere cortical (prefrontal, parietal) and subcortical (striatal, thalamic) nodes gives rise to a pathological bias of attention away from the left side (left-sided inattention) (Robertson and Marshall, 1993). A number of studies of children and adults with ADHD and the biological parents of children with ADHD have shown abnormal biases of spatial attention (Carter et al, 1995; Epstein et al, 1997; Nigg et al, 1997; Sheppard et al, 1999). Most typically, these studies show a subtle rightward bias or left-sided inattention associated with ADHD (Epstein et al, 1997; Sheppard et al, 1999), which is consistent with the predominantly right-hemisphere pathology of the disorder (Casey et al, 1997; Castellanos et al, 1994, 1996; Silk et al, 2005; Sowell et al, 2003).

We have previously reported an association between the 10-repeat DAT1 allele and spatial bias in children and adolescents with ADHD (Bellgrove et al, 2005a,b). Specifically, 10-repeat homozygotes displayed left-sided inattention, relative to 10-repeat heterozygotes (Bellgrove et al, 2005b). We also reported an association between left-sided inattention in ADHD and an enhanced response to MPH: children who were homozygous for the 10-repeat DAT1 allele and were retrospectively rated by their parents as responding favorably to $\mathrm{MPH}$, were more likely to show left-sided impairment of spatial attention than those who were heterozygous for the 10-repeat allele and were rated as achieving a poorer response (Bellgrove et al, 2005b). These studies suggest that spatial bias may be a marker of stimulant response in ADHD, with left-sided inattention being associated with an enhanced response to stimulants. A limitation of this previous work, however, was the recruitment of medication-withdrawn, rather than naive, participants and the use of retrospective ratings of clinical response.

There are a number of good reasons to suspect that lateral biases of spatial attention-potentially mediated by weaker right-hemisphere (striato-parietal) function-might be associated with stimulant response in ADHD. First, pathological biases of attention have been shown to be ameliorated by dopaminergic agents in a number of clinical conditions, including stroke, schizophrenia, and ADHD (Fleet et al, 1987; Maruff et al, 1995; Sheppard et al, 1999). Conversely, spatial biases can be induced by lesioning ascending dopaminergic pathways (Iversen, 1984) or via drugs, such as clonidine, which attenuate catecholamine function (Coull et al, 2001). Second, stimulants are known to act on areas of the brain that are important for directed attention, including the striatum and frontal and parietal cortices (Mehta et al, 2000; Volkow et al, 1998). High densities of DAT-immunoreactive axons have also recently been reported in the parietal lobe (Lewis et al, 2001). Third, an important role for the parietal lobe in ADHD has recently emerged from brain imaging studies. Thus, for example, a number of structural and functional imaging studies have shown prominent abnormalities in right parietal areas (Konrad et al, 2006; Silk et al, 2005; Smith et al, 2006; Sowell et al, 2003; Tamm et al, 2006). Reduced cortical thickness within the right parietal lobe has been reported in children and adults with ADHD (Makris et al, 2007). Shaw et al (2006) reported normalization of cortical thickness within the right parietal lobe over time in those ADHD children with an improved clinical outcome. Moreover, a recent physiological study showed lower regional cerebral blood flow in the right superior parietal lobule of nonresponders after 8 weeks of treatment with MPH (Cho et al, 2006).

These converging lines of evidence lead us to propose that lateral biases of spatial attention may relate to allelic 
variation in DAT1 and have utility as a marker of stimulant response in ADHD.

The aims of the current study were fourfold. First, we examined whether the 10-repeat allele of the DAT1 VNTR was associated with symptom severity in a cohort of 96 Irish families. We predicted that homozygosity for the 10-repeat allele would be associated with greater symptom severity. Association with a potentially functional VNTR within intron 8 of the DAT1 gene, the three-repeat allele of which was recently reported to be associated with ADHD (Brookes et al, 2006) was also examined. Brookes et al (2006) reported association of ADHD with a common haplotype comprising the 10- and 3-repeat alleles of the DAT1 VNTRs. Asherson et al (2007) recently confirmed this haplotype association with an average odds ratio of 1.4 in a multicenter study involving 1159 probands with DSM-IV combined-type ADHD. (It should be noted that Asherson et al identified additional repeat units in the intron 8 VNTR and relabeled the three-repeat allele as a six-repeat allele. The associated haplotype therefore comprised the 10- and 6-repeat alleles of the $3^{\prime}$ UTR and intron 8 VNTRs.) We therefore also examined whether ADHD symptom severity varied as a function of possession of this 'risk haplotype'.

Second, within this Irish cohort, we sought to verify the association between spatial bias in ADHD and genotype at these DAT1 markers. We predicted that homozygosity for the 10-repeat DAT1 allele would be associated with leftsided inattention.

Third, using a prospective pharmacogenetic design with a medication naive cohort $(n=26)$, we examined whether stimulant treatment modified spatial bias, as a function of DAT1 genotype.

Finally, we also examined whether spatial bias measured when ADHD children were stimulant naive, could predict stimulant response at 6 weeks, independent of genotypic influences. We hypothesized that children displaying leftsided inattention at baseline would show an enhanced clinical response to stimulant medication.

\section{MATERIALS AND METHODS}

\section{Participants}

A total of 96 children and adolescents with ADHD (81 male) participated in this study. Data from 43 of these participants has been presented previously (Bellgrove et al, 2005b). A total of 46 of the children were stimulant-medication naive and were recruited as part of a larger pharmacogenetic study. Participants from the pharmacogenetic study were stimulant naive and had not been prescribed any other psychotropic medication. In all other respects, the inclusion, exclusion, and diagnostic criteria were identical for the entire sample of 96 participants (see below). All recruitment was conducted in accordance with the approved ethical guidelines of participating institutions and hospitals. The mean age of the current sample was 10.3 years $(S D=3)$ with a mean IQ of $97(\mathrm{SD}=14)$. Children were also assessed using the reading and spelling subtests of the Wide Range Achievement Test 3 (Wilkinson, 1993). Participants scoring $1 \frac{1}{2}$ SDs below normative levels on the reading subtest were classified as having reading disorder (RD). Handedness was assessed using the Edinburgh Handedness Inventory with $82 \%$ of the sample being right-handed.

Participants were referred to this study either via support groups or collaborating consultant child and adolescent psychiatry teams. For the pharmacogenetic aspect of this study, all children had been referred to consultant child and adolescent psychiatrists in the community. Confirmation of clinical diagnoses were made by trained psychiatrists ( $\mathrm{AK}$, EB) using the parent version of the Child and Adolescent Psychiatric Assessment interview (Angold et al, 1995). Additional information regarding symptom pervasiveness was obtained using The Child Attention-Deficit Hyperactivity Disorder Teacher Telephone Interview (Holmes et al, 2004). All children met DSM-IV diagnosis for ADHD. In this cohort of 96 participants, $74(77 \%)$ of the ADHD participants had a diagnosis of ADHD combined type (ADHD-CT), 12 (13\%) participants had a diagnosis of ADHD predominantly inattentive type (ADHD-In) and 10 (10\%) participants had a diagnosis of ADHD predominantly hyperactive/impulsive type (ADHD-Hyp/Imp). These frequencies did not differ as a function of genotype at the DAT1 markers. Parents of children also rated behavior using either the brief or long forms of the Conners' Parent Rating Scale (Conners, 1997). The mean value for the parent-rated ADHD index was $77(\mathrm{SD}=7.3$; range $=50-91$. (Since a greater range of values was beneficial for correlational analyses using the ADHD index, we did not exclude participants with $T$-scores less than 65, unless otherwise stated. Nevertheless, only 4 participants had ADHD index $T$-scores less than 65 and excluding these participants did not change the association between DAT1 genotype and spatial bias reported.) The frequency of oppositional defiant disorder (ODD) and conduct disorder (CD) within this sample was 51 and 19\%, respectively. Detailed clinical information for the cohort of 96 participants can be found in Table 1 .

\section{Establishing Stimulant Response Using Clinical Ratings}

Predictors of stimulant response were also examined in a subsample of 26 (22 male, 17 right-handed) stimulant-naive children with ADHD (see Table 2). These children met the inclusion criteria for this aspect of the study by having baseline Conners' ADHD index T-scores greater than 65 and having performed the Landmark Task at baseline and then again after 6 weeks of stimulant medication. Additionally, these children did not have a primary diagnosis of pervasive developmental disorder, were free of neurological disorder, and did not have fetal alcohol disorder or prenatal drug abuse. Diagnostic procedures were as described above and $19(73 \%)$ of these children met criteria for ADHD-CT, 4 (15\%) for ADHD-In, and 3 (12\%) for ADHD-Hyp/Imp. The frequency of ODD and CD were 39 and $23 \%$, respectively. Stimulant type and dose was at the discretion of the treating consultant psychiatrist within the community. A total of 25 (96\%) of the ADHD participants were treated with MPHbased preparations (Ritalin (11); Concerta (11); Ritalin LA (3)) and one participant with dextroamphetamine. Dose was calculated as $\mathrm{mg} / \mathrm{kg}$ of MPH equivalent and varied between participants from 0.21 to $1.0 \mathrm{mg} / \mathrm{kg}$ (mean $=0.59 \mathrm{mg} / \mathrm{kg}$, $\mathrm{SD}=0.19)$. MPH equivalent dosages were calculated to facilitate comparison across all types of stimulant medication by 
doubling the dextroamphetamine dose to reach an equivalent MPH-dose (see (Efron et al, 1997)). Dose at 6 weeks was corrected for weight (milligram per kilogram of MPH equivalent).

Table I Clinical Characteristics of ADHD Participants $(n=95)$

\begin{tabular}{llcr}
\hline & Mean & SD & Range \\
\hline Age (years) & 10.3 & 3 & $4-15.8$ \\
IQ & 96 & 14.2 & $71-137$ \\
WRAT reading & 93 & 14 & $55-121$ \\
WRAT spelling & 91 & 15 & $52-149$ \\
ADHD index T-score & 77 & 7.3 & $50-9 \mid$
\end{tabular}

Number (\%)

\begin{tabular}{ll}
\hline Handedness (right-handed) & $79(82)$ \\
Gender (male) & $81(84)$ \\
Reading disorder & $17(18)$ \\
ADHD-CT & $74(77)$ \\
ADHD-In & $12(13)$ \\
ADHD-Hyp/lmp & $10(10)$ \\
Oppositional defiant disorder & $49(51)$ \\
Conduct disorder & $15(16)$
\end{tabular}

Abbreviations: WRAT, wide range achievement test; ADHD-CT, ADHD combined type; ADHD-In, ADHD inattentive type; ADHD-Hyp/lmp, ADHD hyperactive/impulsive type.

WRAT reading and spelling measures were not available for nine participants with ADHD.
In the current analysis, stimulant response was assessed using parental ratings of symptomatology obtained at baseline and then again after 6 weeks of stimulant treatment. Here, we focus on medication-related changes in parent-rated Conners' ADHD indices at baseline and then at 6 weeks. A categorical stimulant-response variable was also defined based upon whether ADHD indices were normalized with stimulant treatment $(T$-scores $\leqslant 60)$ or not $(T>60)$.

\section{Using Spatial Bias as a Marker of Stimulant Response}

Spatial bias, or asymmetry, was assessed using the Landmark Task (Bellgrove et al, 2005b; Binder et al, 1992; Fink et al, 2001). In this brief ( $5 \mathrm{~min})$ test, participants judge which end of a pre-bisected line looks shorter to them. Participants performed 20 trials of the Landmark Task. On 10 of these trials, the bisecting line was offset (either to the right or left) allowing accuracy of judgments to be determined. On the remaining 10 trials, the horizontal line was bisected in the middle. Trials on which the left end of the line was nominated as the shorter were designated 'right-biased'. Trials on which the right end of the line was nominated as the shorter were designated 'left-biased'. A continuous measure of spatial bias - the Landmark Asymmetry index - was calculated as ( $\mathrm{N}_{\text {right-biased }}$ trials $-\mathrm{N}_{\text {left-biased }}$ trials $)$ / 10. This yielded values ranging from -1 (leftward bias) to +1 (rightward bias). Normally developing children tend to show a subtle leftward bias on versions of line bisection giving rise to negative values for asymmetry indices (Dobler et al, 2001; Failla et al, 2003). Human lesion and functional

Table 2 Clinical Characteristics of ADHD Participants Involved in the Medication-Response Study $(n=26)$, as a Function of DATI 3'UTR VNTR Genotype Group

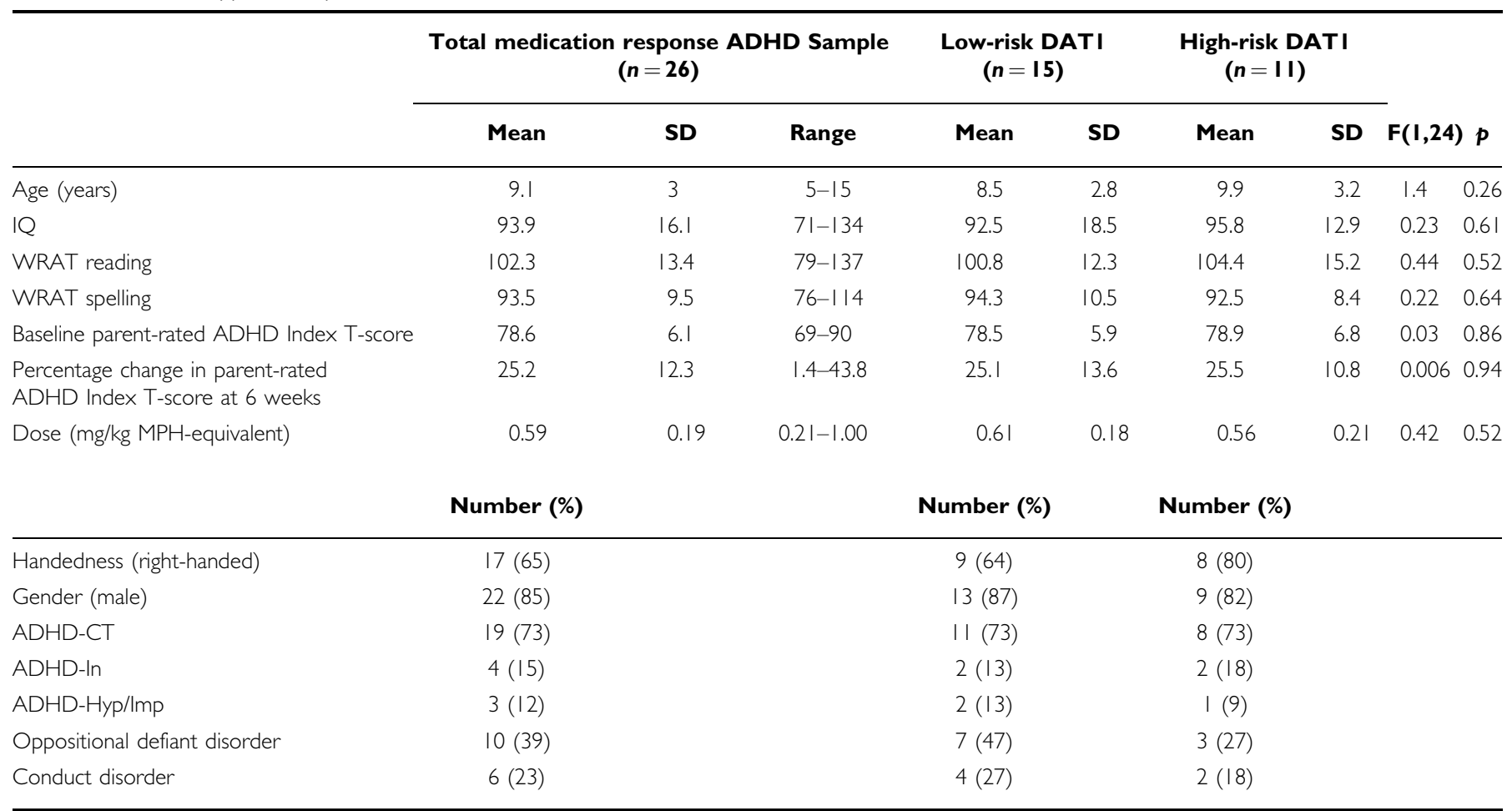

WRAT, wide range achievement test; ADHD-CT, ADHD combined type; ADHD-In, ADHD inattentive type; ADHD-Hyp/lmp, ADHD hyperactive/impulsive type Low-risk DATI: no or one copy of the 10-repeat allele; high-risk DATI: two copies of the 10-repeat allele. 
neuroimaging studies have shown that this task is a particularly sensitive assay of right parietal function (Binder et al, 1992; Fink et al, 2000, 2001). A total of 96 children and adolescents performed the Landmark Task while either medication naive (48\%) or withdrawn from stimulant medication for at least $24 \mathrm{~h}$. A total of 26 of those children who were medication naive also performed the task again after 6 weeks of stimulant treatment. Changes in the Landmark Asymmetry index as a function of medication phase (baseline vs 6 weeks) and DAT1 genotype were of interest, as well as the relationship between baseline Landmark Asymmetry indices and stimulant-response variables.

\section{DAT1 Genotyping}

DNA was extracted from blood samples or saliva using the standard phenol chloroform procedure from both parents and the ADHD proband in each family. Primer sequence and amplification conditions for the $3^{\prime}$-UTR VNTR can be found elsewhere (Daly et al, 1999). Polymerase chain reaction (PCR) amplification of the intron 8 VNTR was performed using the following primers $(10 \mathrm{pmol} / \mu \mathrm{l}$ each): (forward: $5^{\prime}$-GCTTGGGGAAGGAAGGG-3' ; reverse $5^{\prime}$-TGTG TGCGTGCATGTGG-3 $3^{\prime}$ ). The following PCR cycling protocol was adopted: $95^{\circ} \mathrm{C}$ for $15 \mathrm{~min}$, with 30 cycles of annealing $66^{\circ} \mathrm{C}$ for $1 \mathrm{~min}$, and extension $72^{\circ} \mathrm{C}$ for $1 \mathrm{~min}$. A final 10 -min extension at $72^{\circ} \mathrm{C}$ was also added. Amplification products were visualized on $2 \%$ agarose gels, denoting the two common repeats as alleles 2 and 3 at 339 and $369 \mathrm{bp}$, respectively. All genotypes were in Hardy-Weinberg Equilibrium (3'-UTR VNTR: $\chi_{2}^{2}=0.86, p=0.65$; intron 8 VNTR: $\left.\chi_{2}^{2}=0.31, p=0.86\right)$.

Linkage disequilibrium (LD) between the $3^{\prime}$ UTR and intron 8 VNTRs was estimated at $D^{\prime}=0.67$ for the entire sample. DAT1 10/3 haplotype status was assigned to participants using a Bayesian approach to reconstructing haplotypes implemented in the program PHASE (v2.02 Stephens and Donnelly, 2003). Haplotypes could be determined for 90 participants at a probability $\geqslant 0.9$. A total of $46(46 \%)$ participants were homozygous for the 'high-risk' 10/3 haplotype, 38 (42\%) were heterozygous, and $12(12 \%)$ did not possess this haplotype. The frequency of the $10 / 3$ haplotype within this Irish cohort was $66 \%$.

\section{Statistical Analysis}

Statistical analyses of the relationships between DAT1 genotype and both symptom severity and spatial bias were performed using analysis of variance (ANOVA). Participants who were homozygous for risk alleles (10-repeat $3^{\prime}$ UTR VNTR or three-repeat intron 8 VNTR) were classified as 'high-risk'. Participants possessing < two copies of the risk alleles were classified as 'low-risk'. Genotypes at both markers were entered as between-subjects factors in analyses in order to examine the main effect of one in the presence of the other. The effect of increasing possession of the 10/3 DAT1 risk haplotype was also examined using ANOVA with a between-subjects factor of DAT1 haplotype

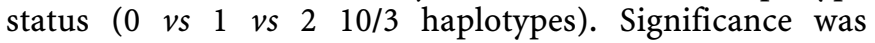
assessed at an $\alpha$ of 0.05 and all post hoc comparisons were Bonferroni corrected.
For the medication-response aspect of this study, all analyses were covaried for IQ and dose (milligram per kilogram of MPH equivalent). The interaction of DAT1 genotype and medication phase (naive $v s \quad 6$ weeks of stimulant treatment) on Landmark Asymmetry indices was assessed using ANOVA, with repeated measures on the Landmark Asymmetry index assessed at baseline and then again after 6 weeks. To examine whether spatial bias predicted stimulant-related symptom reduction after 6 weeks of treatment, the Landmark Asymmetry index was used as a predictor in regression, independent of the influences of IQ and dose. Baseline Landmark Asymmetry indices were compared using ANOVA, as a function of the categorical medication-response variable (normalization vs non-normalization of ADHD indices).

\section{RESULTS}

\section{DAT1 Variants Influence Symptom Severity in ADHD}

The association between DAT1 variants and dimensional measures of ADHD symptomatology was firstly examined using the ADHD index from the Conners' scales. There was a significant main effect of $3^{\prime}$-UTR genotype on the ADHD index $\left(\mathrm{F}(1,92)=5.34, p<0.05, \eta^{2}=0.06\right)$, such that HighRisk group had higher levels of symptomatology (mean $=80, \mathrm{SE}=1.6$ ) than the Low-Risk group (mean $=76$, $\mathrm{SD}=1.1)$. There was no main effect of intron 8 genotype $\left(\mathrm{F}(1,92)=2.25, p>0.05, \eta^{2}=0.02\right)$. A separate univariate ANOVA revealed a significant main effect of DAT1 haplotype status $\left(\mathrm{F}(2,85)=3.12, p<0.05, \eta^{2}=0.07\right)$, after controlling for the influence of age and IQ. ADHD probands who did not possess the $10 / 3$ haplotype had significantly lower symptom levels (mean $=72.5, \mathrm{SE}=2.1$ ) than those who possessed one (mean $=78, \mathrm{SE}=1.1 ; p=0.05$; Bonferroni corrected) or two (mean $=78, \mathrm{SE}=1.1 ; p=0.08) 10 / 3$ haplotypes. Given the absence of an effect for the intron 8 VNTR, the $10 / 3$ haplotype effect is most likely driven by the association with the $3^{\prime}$-UTR VNTR.

\section{DAT1 Variants Influence Spatial Bias in ADHD}

The association between DAT1 variants ( $3^{\prime}$ UTR and intron 8) and spatial bias was next examined in a sample of 96 children and adolescents with ADHD. There was a significant main effect of DAT1 3 '-UTR genotype on spatial bias $\left(\mathrm{F}(1,92)=4.76, p<0.05, \eta^{2}=0.05\right)$, such that the highrisk group were right-biased (mean $=+0.21, \mathrm{SE}=0.09)$ and the low-risk group were left-biased (mean $=-0.03$, $\mathrm{SE}=0.06)$. The main effect of DAT1 intron 8 genotype was not significant $\left(\mathrm{F}(1,92)=2.66, p>0.05, \eta^{2}=0.03\right)$. A separate univariate ANOVA found no main effect of DAT1 haplotype status ( 0 vs 1 vs 2 10/3 haplotypes) on spatial bias $\left(\mathrm{F}(2,87)=0.60, p=0.55, \eta^{2}=0.01\right)$. Restricting the above analysis to children without RD $(n=77 ; \mathrm{F}(1,73)=5.76$, $\left.p<0.05, \eta^{2}=0.07\right)$ or to right-handed children $(n=79$; $\left.\mathrm{F}(1,75)=6.07, p=0.02, \eta^{2}=0.08\right)$ marginally increased the strength of the association between the $3^{\prime}$-UTR marker and spatial bias.

There was a significant, albeit weak, correlation between spatial bias and scores on the ADHD index, indicating that 
greater symptom severity was associated with poorer leftsided attention ( $r=0.19, p<0.05$, one-tailed).

In summary, lateral biases of spatial attention in ADHD appear to be most robustly influenced by variation in the DAT1 $3^{\prime}$ UTR, with less influence of the intron 8 VNTR. ADHD probands with the high-risk DAT1 genotype at the $3^{\prime}$ marker displayed a subtle left-sided inattention/right spatial bias whereas those with the low-risk genotype at this marker did not. Spatial bias was related to the severity of symptomatology in ADHD.

\section{DAT1 Variants Influence the Effect of Stimulant Medications on Spatial Bias in ADHD}

Spatial biases at baseline and after 6 weeks of stimulant medication were examined as a function of DAT1 variants $(n=26)$. Since lower IQ is known to moderate stimulant response (Owens et al, 2003), all analyses reported below were covaried for IQ. Since dose was at the discretion of the treating clinician, all analyses were also covaried for dose. Participant characteristics, as a function of DAT1 $3^{\prime}$-UTR genotype, are presented in Table 2. RD has been associated with both a poorer response to stimulants and left-sided inattention (Facoetti et al, 2001). We therefore excluded children with RD from the current analysis.

Due to the smaller number of participants involved in this aspect of the study, the $3^{\prime}$ UTR and intron 8 markers were analyzed separately. Results are reported for each of these markers along with the 10/3 haplotype.

There was a significant interaction between $3^{\prime}$-UTR genotype and medication phase (baseline vs 6 weeks) on the Landmark Asymmetry index measured at baseline and again at 6 weeks $\left(\mathrm{F}(1,22)=5.03, p<0.05, \eta^{2}=0.19\right)$. Effects were comparable when excluding non right-handed children $\left(\mathrm{F}(1,13)=4.61, p=0.05, \eta^{2}=0.26\right)$. There were no main effects of $3^{\prime}$-UTR genotype $(\mathrm{F}(1,22)=0.08, p>0.05)$, dose $(\mathrm{F}(1,22)=3.69, p>0.05)$, or IQ $(\mathrm{F}(1,22)=0.29, p>0.05)$. Inspection of Figure 1 indicates that lateral biases changed as a function of $3^{\prime}$-UTR genotype and medication phase. The high-risk group were right-biased at baseline (as reported above) and became slightly left-biased with medication. In contrast, the low-risk groups were slightly left-biased at baseline and became right-biased with medication. Analysis of simple main effects with Bonferroni corrections showed the latter effect drove the interaction $(p<0.05)$.

We also separately examined the interaction of medication phase and intron 8 genotype and DAT1 haplotype status (0/1 10/3 haplotype vs $210 / 3$ haplotypes (Due to the smaller sample size in this aspect of the study, individuals who did not possess the 10/3 haplotype were combined with $10 / 3$ heterozygotes and compared to 10/3 homozygotes.)) on the Landmark Asymmetry index. No interaction was found between intron 8 genotype and medication phase $(\mathrm{F}(1,22)=0.05, p>0.05)$.

An interaction was however found between DAT1 haplotype status and medication phase $(\mathrm{F}(1,22)=5.05$, $\left.p<0.05, \eta^{2}=0.19\right)$ that resembled that for the $3^{\prime}$-UTR marker. The effect of stimulant medication on lateral biases of spatial attention was most pronounced in those with none or one 10/3 haplotype ( $p<0.05$, Bonferroni corrected).

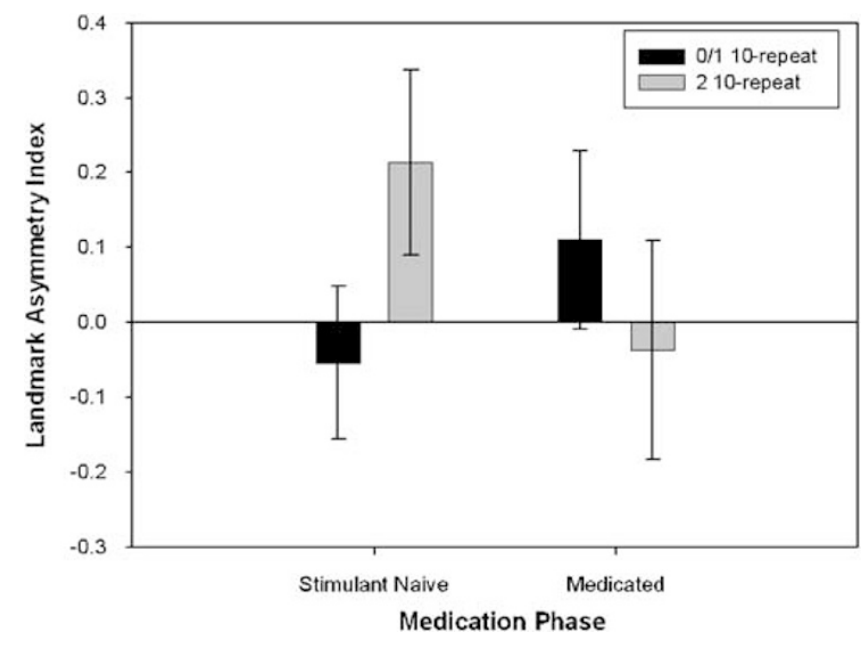

Figure I Interaction of DATI $3^{\prime}-U T R$ genotype and medication phase on the Landmark Asymmetry index (mean \pm I SE).

\section{DAT1 Variants Do Not Influence Stimulant Response in ADHD}

Given reports that the 10-repeat allele of the DAT1 VNTR may mediate an enhanced response to stimulants (Kirley et al, 2003), we examined whether there was a relationship between DAT1 genotype and either the dose of stimulant prescribed (milligram per kilogram of MPH equivalent) or the extent of symptom reduction achieved by 6 weeks of stimulant treatment. There was no significant effect of DAT1 3'-UTR genotype on either dose $(F(1,24)=0.42$, $p=0.52$ ) or the percentage change in parent-rated ADHD index scores $(\mathrm{F}(1,24)=0.006, p=0.94)$. Effects were also nonsignificant for the DAT1 intron 8 VNTR and $10 / 3$ haplotype. Within this cohort, the mean percentage change score in the ADHD index was $25 \%(\mathrm{SD}=12.3)$, with 12 participants (46\%) achieving a symptom reduction of $25 \%$ or more.

\section{Spatial Bias Predicts Stimulant Response}

A linear regression was performed to examine whether the Landmark Asymmetry index measured at baseline could predict the percentage change in parent-rated ADHD index scores after 6 weeks of treatment. The Landmark Asymmetry index was a significant predictor, accounting for $19 \%$ of the variance in the parent-rated ADHD index percentage change scores $(\mathrm{F}(1,24)=5.45, p<0.05)$. Dose of stimulant drug (milligram per kilogram) and IQ were not independent predictors of symptom change.

A categorical medication-response variable was formed by examining whether or not parent-rated ADHD indices normalized with 6 weeks of stimulant treatment. A total of 13 participants normalized (ADHD index $T$-scores $\leqslant 60$ ), whereas 13 did not (ADHD index $T$-scores $>60$ ). There were no baseline differences on the parent-rated ADHD index between these two groups $(\mathrm{F}(1,24)=0.29, p>0.05)$. After controlling for the effects of IQ and dose (milligram per kilogram of MPH equivalent), there was a significant difference between those who did and did not normalize in terms of their baseline Landmark Asymmetry indices $\left(\mathrm{F}(1,22)=4.82, p<0.05, \eta^{2}=0.18\right)$. Those who normalized 
were significantly right-biased at baseline $($ mean $=+0.17$, $\mathrm{SD}=0.28 ; \quad t(12)=2.17, \quad p<0.05$, one-tailed), whereas those who did not tended to be left-biased at baseline $($ mean $=-0.14, \mathrm{SD}=0.46 ; t(12)=1.07, p=0.15$, one-tailed $)$ (see Figure 2).

\section{DISCUSSION}

This study examined the relationship between a neurocognitive phenotype-spatial attentional bias and symptom severity and DAT1 genotype in children and adolescents with ADHD. Using a prospective pharmacogenetic design, we also investigated the utility of spatial bias for predicting the clinical response to stimulants after 6 weeks of treatment. There were several noteworthy findings.

First, variation at the $3^{\prime}$-UTR VNTR associated with ADHD symptom severity, such that 10-repeat homozygotes had higher levels of parent-rated symptomatology than those children possessing none or one copy of this allele. Moreover, an effect of the 10/3 haplotype on symptom severity was apparent, with lower symptom severity in probands who did not possess this haplotype. That we found no influence of the intron 8 VNTR on symptom severity suggests that variation in the $3^{\prime}$ UTR is the primary driver of these relationships within our Irish sample.

Second, the results of the present study support our previous reports of an association between the 10-repeat allele of the DAT1 $3^{\prime}$-UTR VNTR and spatial bias in ADHD (Bellgrove et al, 2005a, b). There was an association between the 10 -repeat allele at the $3^{\prime}$ marker and spatial bias, with left-sided inattention being most pronounced in ADHD probands who were homozygous for the 10-repeat allele. Moreover, left-sided inattention was associated with higher symptom severity.

Third, spatial bias was modulated, as a function of DAT1 genotype, by stimulant treatment. Stimulant treatment influenced spatial biases most dramatically in the low-risk 3 -UTR genotype group where stimulant use induced left-sided inattention. Comparable effects were seen as a function of 10/3 DAT1 haplotype status.

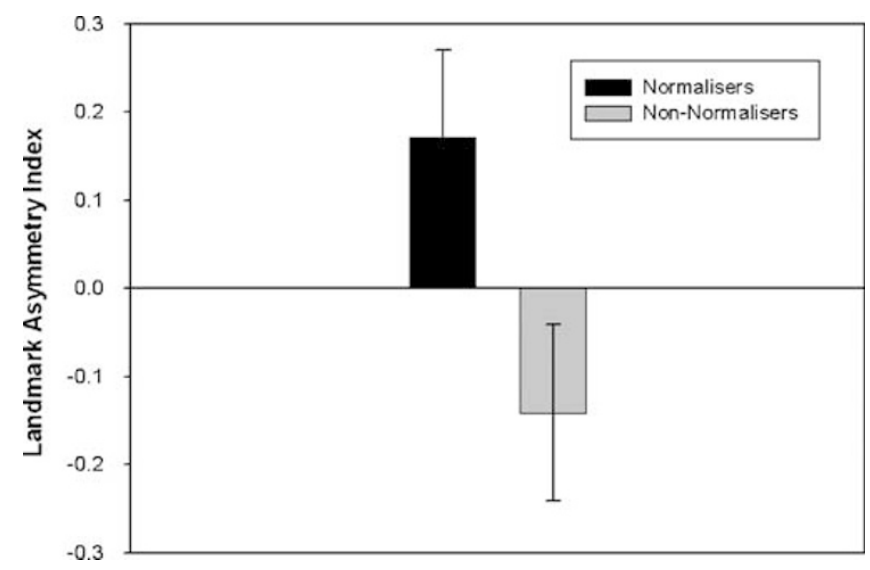

Figure 2 Landmark Asymmetry index predicts stimulant response (mean \pm I SE). ADHD probands who achieved normalization of the parent-rated ADHD index after 6 weeks of stimulant treatment were right-biased (left-sided inattention) at baseline whereas those who did not achieve normalization tended to be left-biased at baseline.
Fourth, irrespective of DAT1 genotype, spatial bias measured at baseline was predictive of the clinical response achieved by 6 weeks of stimulant treatment. Children who achieved a better clinical response to stimulants displayed left-sided inattention when medication naive, whereas those who achieved a poorer response showed the opposite bias. This result suggests that left-sided inattention measured at baseline is a predictor of an enhanced response to stimulant medication in ADHD.

Bellgrove et al (2005b) reported an association between left-sided inattention and the 10-repeat allele of the DAT1 3 -UTR VNTR in a sample of 43 ADHD probands who were free of RD. Here, we extend these findings in a partially overlapping sample to show a general effect of DAT1 genotype on spatial bias in an unselected sample of 96 participants with $\mathrm{ADHD}$. In contrast to the left-sided inattention displayed by the high-risk group, the spatial bias of the low-risk group was consistent with that displayed by healthy participants. Healthy participants performing line bisection, on average, tend to bisect lines slightly to the left of the objective center (Bowers and Heilman, 1980). This subtle leftward bias is thought to arise from the dominance of the right-hemisphere for spatial judgments. The bias displayed by the high-risk group is, however, opposite in direction and is comparable - in a subclinical form - to that seen after right hemisphere lesions to frontal, parietal, or subcortical regions (Robertson and Marshall, 1993). Leftsided inattention on the Landmark Task has been particularly associated with damage to the right parietal lobe (Binder et al, 1992). Support for the association between DAT1 genotype and spatial bias in ADHD comes from work showing evidence of high densities of DATimmunoreactive axons in the parietal lobe (Lewis et al, 2001). Given accumulating evidence suggesting functionality of the $3^{\prime}$-UTR VNTR, we have hypothesized that the 10-repeat DAT1 allele, or another variant with which it is in LD, might be associated with lowered dopamine in righthemisphere spatial attention networks (eg striatum and parietal lobe), thus giving rise to left-sided inattention (Bellgrove et al, 2005b).

It is noteworthy that in the current study, we found no influence of the intron 8 VNTR or of the 10/3 DAT1 haplotype on spatial bias. This was somewhat surprising given that the $3^{\prime}$ UTR and intron 8 VNTRs show a considerable degree of LD within our Irish sample and we have previously shown an effect of the intron 8 VNTR and $10 / 3$ haplotype on spatial orienting in healthy children (Bellgrove et al, 2007). Spatial orienting paradigms examine the influence of spatial cues that are either valid, invalid, or uninformative with respect to the hemifield of an upcoming target. Over a large number of trials orienting tasks yield reaction time effects that reflect the operation of spatial selective attention. These tasks may have greater sensitivity to detect subtle genetic influences than the Landmark Task which probes directed attention over a relatively small number of trials. We cannot therefore discount an influence of the intron 8 VNTR or 10/3 haplotype on spatial attention in ADHD. Nevertheless, the current study provides additional evidence for an association between the 10-repeat allele of the DAT1 $3^{\prime}$-UTR VNTR and spatial bias in ADHD.

Lateral biases of attention were also modified with stimulant medication differentially in each of the low- and 
high-risk DAT1 3 '-UTR VNTR groups, and as a function of 10/3 haplotype status. Previous work in ADHD has shown that spatial biases can be modified with stimulant treatment (Nigg et al, 1997; Sheppard et al, 1999), but to our knowledge, this is the first report to indicate that this effect is further modified by DAT1 genotype. The spatial bias of the high-risk group shifted with medication from being right-biased (left-sided inattention) at baseline to left-biased when medicated. Stimulant medication had the most profound effect, however, on lateral biases in the low-risk group with a significant rightward shift in spatial bias occurring with treatment. These results suggest that stimulant medication actually induced a pathological leftsided inattention in the low-risk group.

A primary aim of this study was to examine whether lateral biases of spatial attention measured at baseline could predict the clinical response to stimulants after 6 weeks of treatment, independent of genetic influences. A robust relationship was found between spatial bias and medication-related changes in parent-reported ADHD index $T$-scores at 6 weeks compared to baseline (19\% variance explained). ADHD children who achieved greater symptom reduction displayed left-sided inattention at baseline, after controlling for the influence of IQ and stimulant dose. This relationship held when classifying clinical response according to whether ADHD symptoms were normalized with treatment or not. Our results demonstrate that lateral biases of attention have efficacy in predicting clinical response to stimulants, irrespective of genotypic influences. This result supports our previously published finding that spatial bias was related to retrospective ratings of MPH response in ADHD (Bellgrove et al, 2005b). Importantly, the current result cannot be attributed to prior exposure to stimulant medications, differential baseline symptom levels, or the dose required to achieve symptom reduction.

A number of studies have examined whether the 10repeat DAT1 allele is a significant predictor of stimulant response, however, results have proved conflicting (Cheon et al, 2005; Kirley et al, 2003; Rohde et al, 2003; Roman et al, 2002; Stein et al, 2005; Winsberg and Comings, 1999). Kirley et al (2003) previously reported an association between the 10-repeat allele and an enhanced response to MPH using a three-point retrospective rating-scale within an Irish population. The present study used a prospective design to examine this issue in a relatively small sample of 26 medication naive children with ADHD but failed to find an effect of DAT1 genotype on the percentage change score for the ADHD index after 6 weeks of stimulant treatment. It should be noted, however, that the current sample of 26 children had considerably reduced power to detect this effect.

In summary, the results of the present study suggest that spatial bias may be a marker of symptom severity, genetic risk, and stimulant response in ADHD. The current study supports our previous report (Bellgrove et al, 2005b) of an association between spatial bias and both symptom severity and the 10-repeat allele of the DAT1 3 '-UTR VNTR in our Irish ADHD sample. In the absence of definitive data linking VNTR alleles to DAT expression, these data nevertheless show a functional effect of the 10-repeat allele on directed attention in ADHD. Our results also show that stimulant medications modulate spatial bias differentially as a function of DAT1 genotype; 6 weeks of stimulant treatment induced left-sided inattention in the low-risk DAT1 group and attenuated this phenotype in the high-risk DAT1 group. This effect implies that allelic variation in DAT1 differentially influences the sensitivity of spatial attention networks to stimulant drugs. The association between left-sided inattention at baseline and a greater symptom reduction after 6 weeks of stimulant treatment is also an important and novel finding. If confirmed within larger prospective studies, baseline assessments of spatial bias, irrespective of genotype status, may have clinical utility for predicting response to stimulants in ADHD.

\section{ACKNOWLEDGEMENTS}

This work was supported by grants from the Irish Health Research Board, Science Foundation Ireland, the Irish Higher Education Authority's Programme for Research in Third-Level Institutions. MAB is supported by an Australian National Health and Medical Research Council Howard Florey Centenary Fellowship. KAJ is supported by the Health Research Board of Ireland. We thank the referring child and adolescent psychiatrists from the Health Service Executive, South Western, North Eastern, and Midlands areas and the Lucena clinics. We also thank the participating children and their families.

\section{DISCLOSURE/CONFLICT OF INTEREST}

Dr Bellgrove, Dr Gill, and Dr Robertson hold a patent within Ireland (Irish Patent no: 84530) on using molecular genetic and attentional markers to predict methylphenidate response in ADHD. The other authors have nothing to disclose.

\section{REFERENCES}

Angold A, Predergast M, Cox A, Harrington R, Simonoff E, Rutter M (1995). The Child and Adolescent Psychiatric Assessment (CAPA). Psychol Med 25: 739-753.

Asherson P, Brookes K, Franke B, Chen W, Gill M, Ebstein RP et al (2007). Confirmation that a specific haplotype of the dopamine transporter gene is associated with combined-type ADHD. Am J Psychiatry 164: 674-677.

Bellgrove MA, Chambers CD, Johnson KJ, Daibhis A, Daly M, Hawi Z et al (2007). Dopaminergic genotype biases spatial attention in healthy children. Mol Psychiatry 12: 786-792.

Bellgrove MA, Gill M, Hawi Z, Kirley A, Robertson IH (2005a). Dissecting the attention deficit hyperactivity disorder (ADHD) phenotype: sustained attention, response variability and spatial attentional asymmetries in relation to dopamine transporter (DAT1) genotype. Neuropsychologia 43: 1847-1857.

Bellgrove MA, Hawi Z, Kirley A, Fitzgerald M, Gill M, Robertson IH (2005b). Association between dopamine transporter (DAT1) genotype, left-sided inattention, and an enhanced response to methylphenidate in attention-deficit hyperactivity disorder. Neuropsychopharmacology 30: 2290-2297.

Binder J, Marshall R, Lazar R, Benjamin J, Mohr JP (1992). Distinct syndromes of hemineglect. Arch Neurol 49: 1187-1194.

Bowers D, Heilman KM (1980). Pseudoneglect: effects of hemispace on a tactile line bisection task. Neuropsychologia 18: 491-498. 
Bradshaw JL (1989). Hemispheric Specialisation and Psychological Function. Wiley: New York.

Brookes KJ, Mill J, Guindalini C, Curran S, Xu X, Knight J et al (2006). A common haplotype of the dopamine transporter gene associated with attention-deficit/hyperactivity disorder and interacting with maternal use of alcohol during pregnancy. Arch Gen Psychiatry 63: 74-81.

Carter CS, Krener P, Chaderjian M, Northcutt C, Wolfe V (1995). Asymmetrical visual-spatial attentional performance in ADHD: evidence for a right hemispheric deficit. Biol Psychiatry 37: 789-797.

Casey BJ, Castellanos FX, Giedd JN, Marsh WL, Hamburger SD, Schubert $\mathrm{AB}$ et al (1997). Implication of right frontostriatal circuitry in response inhibition and attention-deficit/hyperactivity disorder. J Am Acad Child Adolesc Psychiatry 36: 374-383.

Castellanos FX, Giedd JN, Eckburg P, Marsh WL, Vaituzis AC, Kaysen D et al (1994). Quantitative morphology of the caudate nucleus in attention deficit hyperactivity disorder. $A m \mathrm{~J}$ Psychiatry 151: 1791-1796.

Castellanos FX, Giedd JN, Marsh WL, Hamburger SD, Vaituzis AC, Dickstein DP et al (1996). Quantitative brain magnetic resonance imaging in attention-deficit hyperactivity disorder. Arch Gen Psychiatry 53: 607-616.

Cheon KA, Ryu YH, Kim JW, Cho DY (2005). The homozygosity for 10-repeat allele at dopamine transporter gene and dopamine transporter density in Korean children with attention deficit hyperactivity disorder: relating to treatment response to methylphenidate. Eur Neuropsychopharmacol 15: 95-101.

Cheon KA, Ryu YH, Kim YK, Namkoong K, Kim CH, Lee JD (2003). Dopamine transporter density in the basal ganglia assessed with [123I]IPT SPET in children with attention deficit hyperactivity disorder. Eur J Nucl Med Mol Imaging 30: 306-311.

Cho SC, Hwang JW, Kim BN, Lee HY, Kim HW, Lee JS et al (2006). The relationship between regional cerebral blood flow and response to methylphenidate in children with attention-deficit hyperactivity disorder: comparison between non-responders to methylphenidate and responders. J Psychiatr Res 41: 459-465.

Conners CK (1997). Conners' Rating Scales-Revised: Technical Manual. Multi-Health Systems Inc.: New York.

Cook EH, Stein MA, Krasowski MD, Cox NJ, Olkon DM, Kieffer JE et al (1995). Association of attention-deficit disorder and the dopamine transporter gene. Am J Hum Genet 56: 993-998.

Coull JT, Nobre AC, Frith CD (2001). The noradrenergic alpha2 agonist clonidine modulates behavioural and neuroanatomical correlates of human attentional orienting and alerting. Cereb Cortex 11: 73-84.

Daly G, Hawi Z, Fitzgerald M, Gill M (1999). Mapping susceptibility loci in attention deficit hyperactivity disorder: preferential transmission of parental alleles at DAT1, DBH and DRD5 to affected children. Mol Psychiatry 4: 192-196.

Dobler V, Manly T, Atkinson J, Wilson BA, Ioannou K, Robertson IH (2001). Interaction of hand use and spatial selective attention in children. Neuropsychologia 39: 1055-1064.

Dougherty DD, Bonab AA, Spencer TJ, Rauch SL, Madras BK, Fischman AJ (1999). Dopamine transporter density in patients with attention deficit hyperactivity disorder. Lancet 354: 2132-2133.

Dresel S, Krause J, Krause KH, LaFougere C, Brinkbaumer K, Kung HF et al (2000). Attention deficit hyperactivity disorder: binding of [99mTc]TRODAT-1 to the dopamine transporter before and after methylphenidate treatment. Eur J Nucl Med 27: $1518-1524$

Efron D, Jarman F, Barker M (1997). Methylphenidate $v s$ dexamphetamine in children with attention deficit hyperactivity disorder: a double-blind, crossover trial. Pediatrics 100: E6.

Epstein JN, Conners CK, Erhardt D, March JS, Swanson JM (1997). Asymmetrical hemispheric control of visual-spatial attention in adults with attention deficit hyperactivity disorder. Neuropsychology 11: 467-473.
Facoetti A, Turatto M, Lorusso ML, Mascetti GG (2001). Orienting of visual attention in dyslexia: evidence for asymmetric hemispheric control of attention. Exp Brain Res 138: 46-53.

Failla CV, Sheppard DM, Bradshaw JL (2003). Age and respondinghand related changes in performance of neurologically normal subjects on the line-bisection and chimeric-faces tasks. Brain Cogn 52: 353-363.

Fink GR, Marshall JC, Shah NJ, Weiss PH, Halligan PW, GrosseRuyken $\mathrm{M}$ et al (2000). Line bisection judgments implicate right parietal cortex and cerebellum as assessed by fMRI. Neurology 54: $1324-1331$.

Fink GR, Marshall JC, Weiss PH, Zilles K (2001). The neural basis of vertical and horizontal line bisection judgments: an fMRI study of normal volunteers. Neuroimage 14(1 Part 2): S59-S67.

Fleet WS, Valenstein E, Watson RT, Heilman KM (1987). Dopamine agonist therapy for neglect in humans. Neurology 37: $1765-1770$.

Fuke S, Suo S, Takahashi N, Koike H, Sasagawa N, Ishiura S (2001). The VNTR polymorphism of the human dopamine transporter (DAT1) gene affects gene expression. Pharmacogenomics $J$ 1: 152-156.

Gilbert DL, Wang Z, Sallee FR, Ridel KR, Merhar S, Zhang J et al (2006). Dopamine transporter genotype influences the physiological response to medication in ADHD. Brain 129(Part 8): 2038-2046.

Gill M, Daly G, Heron S, Hawi Z, Fitzgerald M (1997). Confirmation of association between attention deficit hyperactivity disorder and a dopamine transporter polymorphism. Mol Psychiatry 2: 311-313.

Heinz A, Goldman D, Jones DW, Palmour R, Hommer D, Gorey JG et al (2000). Genotype influences in vivo dopamine transporter availability in human striatum. Neuropsychopharmacology 22 133-139.

Holmes J, Lawson D, Langley K, Fitzpatrick H, Trumper A, Pay H et al (2004). The Child Attention-Deficit Hyperactivity Disorder Teacher Telephone Interview (CHATTI): reliability and validity. Br J Psychiatry 184: 74-78.

Iversen SD (1984). Behavioural effects of manipulation of basal ganglia neurotransmitters. Ciba Found Symp 107: 183-200.

Jucaite A, Fernell E, Halldin C, Forssberg H, Farde L (2005). Reduced midbrain dopamine transporter binding in male adolescents with attention-deficit/hyperactivity disorder: association between striatal dopamine markers and motor hyperactivity. Biol Psychiatry 57: 229-238.

Kirley A, Lowe N, Hawi Z, Mullins C, Daly G, Waldman I et al (2003). Association of the $480 \mathrm{bp}$ DAT1 allele with methylphenidate response in a sample of Irish children with ADHD. Am J Med Genet 121B: 50-54.

Konrad K, Neufang S, Hanisch C, Fink GR, Herpertz-Dahlmann B (2006). Dysfunctional attentional networks in children with attention deficit/hyperactivity disorder: evidence from an eventrelated functional magnetic resonance imaging study. Biol Psychiatry 59: 643-651.

Krause KH, Dresel SH, Krause J, Kung HF, Tatsch K (2000). Increased striatal dopamine transporter in adult patients with attention deficit hyperactivity disorder: effects of methylphenidate as measures by single photon emission computed tomography. Neurosci Lett 285: 107-110.

Langley K, Turic D, Peirce TR, Mills S, Van Den Bree MB, Owen MJ et al (2005). No support for association between the dopamine transporter (DAT1) gene and ADHD. Am J Med Genet B Neuropsychiatr Genet 139: 7-10.

Lewis DA, Melchitzky DS, Sesack SR, Whitehead RE, Auh S, Sampson A (2001). Dopamine transporter immunoreactivity in monkey cerebral cortex: regional, laminar, and ultrastructural localization. J Comp Neurol 432: 119-136.

Loo SK, Specter E, Smolen A, Hopfer C, Teale PD, Reite ML (2003). Functional effects of the DAT1 polymorphism on 
EEG measures in ADHD. J Am Acad Child Adolesc Psychiatry 42: 986-993.

Makris N, Biederman J, Valera EM, Bush G, Kaiser J, Kennedy DN et al (2007). Cortical thinning of the attention and executive function networks in adults with attention-deficit/hyperactivity disorder. Cereb Cortex 17: 1364-1375.

Maruff P, Hay D, Malone V, Currie J (1995). Asymmetries in the covert orienting of visual spatial attention in schizophrenia. Neuropsychologia 33: 1205-1223.

Mehta MA, Owen AM, Sahakian BJ, Mavaddat N, Pickard JD, Robbins TW (2000). Methylphenidate enhances working memory by modulating discrete frontal and parietal lobe regions in the human brain. J Neurosci 20: RC65.

Mesulam M-M (1981). A cortical network for directed attention and unilateral neglect. Ann Neurol 10: 309-325.

Mick E, Biederman J, Spencer T, Faraone SV, Sklar P (2006). Absence of association with DAT1 polymorphism and response to methylphenidate in a sample of adults with ADHD. Am J Med Genet B Neuropsychiatr Genet 141: 890-894.

Nigg JT, Swanson JM, Hinshaw SP (1997). Covert visual spatial attention in boys with attention deficit hyperactivity disorder: lateral effects, methylphenidate response, and results for parents. Neuropsychologia 35: 165-176.

Owens EB, Hinshaw SP, Kraemer HC, Arnold LE, Abikoff HB, Cantwell DP et al (2003). Which treatment for whom for ADHD? Moderators of treatment response in the MTA. J Consult Clin Psychol 71: 540-552.

Robertson IH, Marshall JC (eds). (1993). Unilateral Neglect: Clinical and Experimental Studies. Lawrence Erlbaum and Associates: Hillsdale, NJ.

Rohde LA, Roman T, Szobot C, Cunha RD, Hutz MH, Biederman J (2003). Dopamine transporter gene, response to methylphenidate and cerebral blood flow in attention-deficit/hyperactivity disorder: a pilot study. Synapse 48: 87-89.

Roman T, Szobot C, Martins S, Biederman J, Rohde LA, Hutz MH (2002). Dopamine transporter gene and response to methlphenidate in attention-deficit/hyperactivity disorder. Pharmacogenetics 12: 497-499.

Shaw P, Lerch J, Greenstein D, Sharp W, Clasen L, Evans A et al (2006). Longitudinal mapping of cortical thickness and clinical outcome in children and adolescents with attention-deficit/ hyperactivity disorder. Arch Gen Psychiatry 63: 540-549.

Sheppard DM, Bradshaw JL, Mattingley JB, Lee P (1999). Effects of stimulant medication on the lateralisation of line bisection judgements of children with attention deficit hyperactivity disorder. J Neurol, Neurosurg Psychiatry 66: 57-63.

Silk T, Vance A, Rinehart N, Egan G, O’Boyle M, Bradshaw JL et al (2005). Decreased fronto-parietal activation in attention deficit hyperactivity disorder, combined type (ADHD-CT): an fMRI study. Br J Psychiatry 187: 282-283.
Smith AB, Taylor E, Brammer M, Toone B, Rubia K (2006). Task-specific hypoactivation in prefrontal and temporoparietal brain regions during motor inhibition and task switching in medication-naive children and adolescents with attention deficit hyperactivity disorder. Am J Psychiatry 163: 1044-1051.

Sowell ER, Thompson PM, Welcome SE, Henkenius AL, Toga AW, Peterson BS (2003). Cortical abnormalities in children and adolescents with attention-deficit hyperactivity disorder. Lancet 362: 1699-1707.

Spencer TJ, Biederman J, Madras BK, Dougherty DD, Bonab AA, Livni $\mathrm{E}$ et al (2007). Further evidence of dopamine transporter dysregulation in ADHD: a Controlled PET Imaging Study Using Altropane. Biol Psychiatry 62: 1059-1061.

Stein MA, Waldman ID, Sarampote CS, Seymour KE, Robb AS, Conlon C et al (2005). Dopamine transporter genotype and methylphenidate dose response in children with ADHD. Neuropsychopharmacology 30: 1374-1382.

Stephens M, Donnelly P (2003). A comparison of bayesian methods for haplotype reconstruction from population genotype data. Am J Hum Genet 73: 1162-1169.

Tamm L, Menon V, Reiss AL (2006). Parietal attentional system aberrations during target detection in adolescents with attention deficit hyperactivity disorder: event-related fMRI evidence. Am J Psychiatry 163: 1033-1043.

van der Meulen EM, Bakker SC, Pauls DL, Oteman N, Kruitwagen CL, Pearson PL et al (2005). High sibling correlation on methylphenidate response but no association with DAT1-10R homozygosity in Dutch sibpairs with ADHD. J Child Psychol Psychiatry 46: 1074-1080.

VanNess SH, Owens MJ, Kilts CD (2005). The variable number of tandem repeats element in DAT1 regulates in vitro dopamine transporter density. BMC Genet 6: 55.

Vles JS, Feron FJ, Hendriksen JG, Jolles J, van Kroonenburgh MJ, Weber WE (2003). Methylphenidate down-regulates the dopamine receptor and transporter system in children with attention deficit hyperkinetic disorder (ADHD). Neuropediatrics 34: 77-80.

Volkow ND, Wang GJ, Fowler JS, Gatley SJ, Logan J, Ding YS et al (1998). Dopamine transporter occupancies in the human brain induced by therapeutic doses of oral methylphenidate. $\mathrm{Am} \mathrm{J}$ Psychiatry 155: 1325-1331.

Volkow ND, Wang GJ, Newcorn J, Fowler JS, Telang F, Solanto MV et al (2007). Brain dopamine transporter levels in treatment and drug naive adults with ADHD. Neuroimage 34: $1182-1190$

Wilkinson GS (1993). The Wide Range Achievement Test (WRAT3). Wide Range Inc.: Wilmington, Delaware, USA.

Winsberg BG, Comings DE (1999). Association of the dopamine transporter gene (DAT1) with poor methylphenidate response. J Am Acad Child Adolesc Psychiatry 38: 1474-1477. 